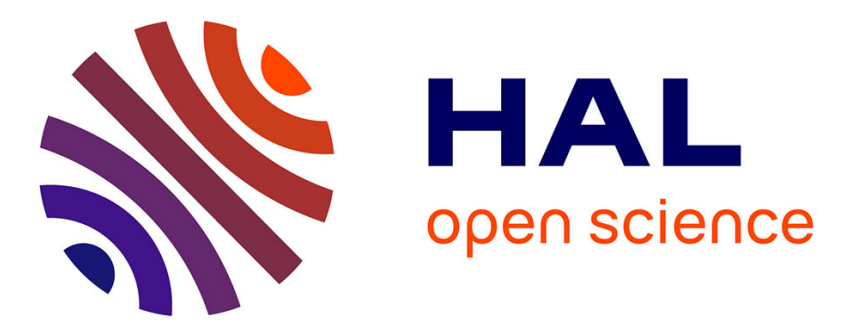

\title{
1968-2018: quatre surprises démographiques en France depuis cinquante ans
}

Gilles Pison

\section{To cite this version:}

Gilles Pison. 1968-2018: quatre surprises démographiques en France depuis cinquante ans. Population et sociétés, 2018, 553, pp.1-4. 10.3917/popsoc.553.0001 . hal-02406979

\section{HAL Id: hal-02406979 \\ https://hal.science/hal-02406979}

Submitted on 12 Dec 2019

HAL is a multi-disciplinary open access archive for the deposit and dissemination of scientific research documents, whether they are published or not. The documents may come from teaching and research institutions in France or abroad, or from public or private research centers.
L'archive ouverte pluridisciplinaire HAL, est destinée au dépôt et à la diffusion de documents scientifiques de niveau recherche, publiés ou non, émanant des établissements d'enseignement et de recherche français ou étrangers, des laboratoires publics ou privés.

\section{()ㅜ) $\Theta$}

Distributed under a Creative Commons Attribution - NoDerivatives| 4.0 International 


\title{
Population \& Sociétés
}

\section{8-2018 : quatre surprises démographiques en France depuis cinquante ans}

\author{
Gilles Pison*
}

\begin{abstract}
La démographie française est faite de continuité et de surprises. À l'occasion des 50 ans de Population et Sociétés (le numéro 1 est paru deux mois avant mai 1968), Gilles Pison attire notre attention sur quatre changements démographiques qui n'avaient pas été anticipés il y a un demisiècle : l'étonnante progression de l'espérance de vie, le retard des maternités, l'augmentation des naissances hors mariage, l'invention et le succès du pacs.
\end{abstract}

La population de la France métropolitaine a franchi le seuil de 50 millions d'habitants il y a exactement 50 ans (en 1968). Elle a augmenté de près d'un tiers depuis et atteint 65 millions en 2018 [1] (tableau et encadré). Sa croissance, comme son vieillissement, avaient été prévus. En revanche, quatre changements démographiques survenus au cours des cinquante dernières années ont été des surprises.

\section{Un accroissement formidable de l'espérance de vie}

L'espérance de vie a augmenté de 11 ans au cours d'un demi-siècle, passant de 71,5 ans sexes confondus en 1967 à 82,5 ans en 2017. Elle progresse moins vite depuis quelques années et n'a gagné que deux mois par an depuis 2010, contre trois en moyenne au cours des cinquante dernières années, laissant penser que l'allongement de la vie pourrait bientôt atteindre ses limites. Si l'on replace ce ralentissement dans le cadre de l'évolution de longue durée, depuis 1950, il apparaît qu'un phénomène similaire de ralentissement des progrès se soit également produit il y a 50 ans (figure 1). Alors que l'espérance de vie à la naissance sexes confondus a augmenté de 4 ans au cours de la décennie 1950, passant de 66,4 ans en 1950 à 70,4 ans en 1960, elle n'a crû que de 1,7 an au cours de la décennie 1960, pour atteindre 72,1 ans en 1970.

Durant cette période, la progression de l'espérance de vie vient encore pour partie du recul de la mortalité infantile. Elle a baissé de moitié entre 1950 et 1960, passant de 51 décès d'enfants de moins d'un an pour mille naissances à $27 \%$ o, et a continué de diminuer pendant la

* Muséum national d'histoire naturelle et Institut national d'études démographiques. décennie suivante pour atteindre $18 \%$ en 1970 . Mais le niveau atteint est si bas qu'elle ne représente plus désormais qu'une faible part de la mortalité, et même si son recul se poursuit, il n'a pratiquement plus d'effet sur l'espérance de vie. Celle-ci ne peut progresser qu'en raison de succès rencontrés dans la lutte contre la mortalité adulte, en particulier aux âges élevés où se concentrent de plus en plus les décès.

Les possibilités de diminution de la mortalité à ces âges ne peuvent être que limitées, pense-t-on à l'époque, et l'espérance de vie va rapidement buter sur un plafond biologique. Le ralentissement des années 1960 vient conforter cette vision. Celle-ci se reflète dans les scénarios d'évolution de l'espérance de vie qu'élabore l'Insee pour projeter la population de la France (figure 1). La projection publiée en 1970, dont l'horizon était 1995, prolonge la tendance à la hausse de l'espérance de vie, d'abord au même rythme que dans la décennie 1960, puis à un rythme décroissant. La projection suivante, publiée en 1979, est plus pessimiste et considère que l'espérance de vie va progresser plus lentement encore, puis plafonner à 73,8 ans en 2000 , sexes confondus. Ces deux projections se sont révélées très en deçà de l'évolution réelle. Le plafond fixé par la seconde projection a été dépassé l'année même où elle a été publiée, l'espérance de vie ayant atteint 74,1 ans en 1979 . Tenant compte de l'énorme décalage entre les projections précédentes et la réalité, la projection de 1986 fait progresser l'espérance de vie nettement plus rapidement tout en conservant l'hypothèse d'un ralentissement puis d'un plafonnement à terme.

Malgré ce relèvement, l'espérance de vie progresse à nouveau plus rapidement que la projection, et le plafond 
Figure 1. Évolution de l'espérance de vie à la naissance en France - observations et projections Ans

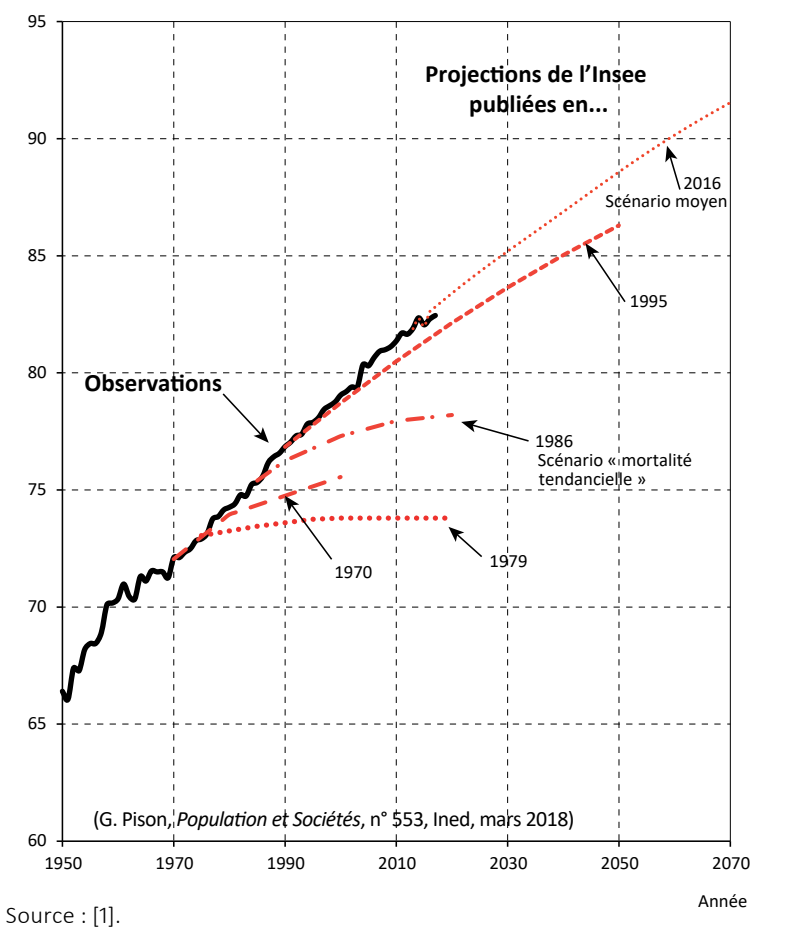

Figure 2. Évolution de l'espérance de vie à 60 ans en France de 1806 à 2017 Ans

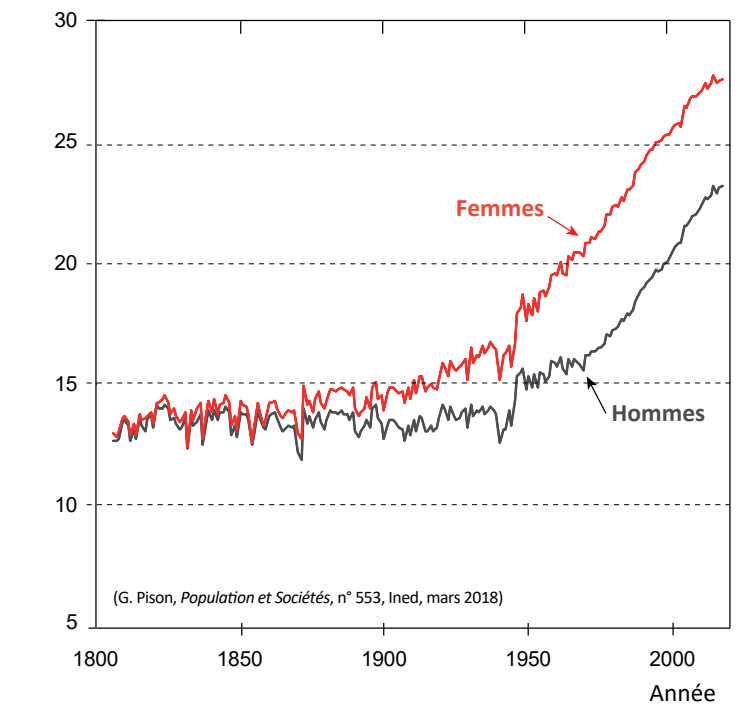

Sources : $[1,4]$.

est atteint puis dépassé en 1997. Le constat que les plafonds même rehaussés sont régulièrement dépassés au bout de quelques années conduit à l'abandon de l'idée même de plafonnement dans les projections suivantes. Celles-ci extrapolent la tendance courante sur toute la période de projection, se contentant de l'infléchir très progressivement sans toutefois fixer de limite. Elles correspondent assez bien à l'évolution observée pour l'instant.
Une surprise : les succès de la lutte contre la mort des adultes et des personnes âgées

Si les projections ont longtemps sous-estimé les progrès de l'espérance de vie, c'est qu'elles n'ont pas anticipé la forte baisse de la mortalité des adultes et des personnes âgées. Il faut dire que les progrès dans ce domaine sont relativement récents, comme le montre l'évolution de l'espérance de vie à 60 ans (figure 2). Au milieu du $\mathrm{XX}^{\mathrm{e}}$ siècle, elle était encore proche de son niveau du XIX ${ }^{\mathrm{e}}$ siècle, notamment du côté masculin : un homme de 60 ans pouvait espérer vivre encore 13 à 14 ans. Ce n'est qu'à partir de la fin de la Deuxième Guerre mondiale qu'elle commence à augmenter chez les hommes, les progrès s'accélérant ensuite jusqu'à ce qu'elle atteigne 23,2 ans en 2014 , soit 7 ans de plus qu'en 1964. La progression a commencé plus tôt chez les femmes, dès les premières décennies du $\mathrm{XX}^{\mathrm{e}}$ siècle, et elle s'est aussi accélérée à partir de la fin de la guerre jusqu'à 27,7 ans en 2014, soit 7,4 ans de plus qu'en 1964. Au milieu du XXe siècle, les maladies infectieuses étaient encore la cause d'une partie importante des décès d'adultes et de personnes âgées, et leur recul a entraîné une augmentation sensible de l'espérance de vie à 60 ans. Mais, comme pour les enfants, la part de ces maladies dans la mortalité totale a beaucoup régressé [2] et les gains à attendre de la poursuite de leur recul sont faibles. Les maladies cardiovasculaires et les cancers sont désormais les principales causes de décès à ces âges. Et ce sont les succès rencontrés dans la lutte contre ces maladies qui ont permis à la mortalité des adultes et des personnes âgées de poursuivre sa baisse à partir des années 1970, et à l'espérance de vie de continuer à augmenter [3]. La mortalité due aux maladies du cœur et des vaisseaux a énormément diminué depuis un demi-siècle grâce aux progrès de la prévention et des traitements. Quant à la mortalité par cancer qui avait augmenté, elle régresse maintenant grâce aux diagnostics plus précoces et à la réduction des comportements à risques.

L'espérance de vie à 60 ans n'a cependant plus progressé au cours des trois dernières années : elle se maintient à 23,2 ans pour les hommes en 2017 comme en 2014, et baisse légèrement pour les femmes $(27,7$ ans en 2014, 27,6 ans en 2017). Même s'il est encore trop tôt pour conclure à un arrêt des progrès, il est possible qu'une nouvelle phase ait débuté dans laquelle l'espérance de vie à 60 ans augmenterait moins vite, notamment chez les femmes.

\section{Autant d'enfants qu'il y a cinquante ans, mais à des âges plus élevés}

Depuis la fin du baby-boom il y a cinquante ans, les femmes mettent au monde environ deux enfants chacune en moyenne en France, quelle que soit la génération. Celles qui ont fêté leurs quarante ans l’an passé, nées en 1977, ont 
déjà 1,99 enfant, et devraient probablement en avoir autour de 2,05 à 50 ans. Leurs mères, nées pour la plupart à la fin des années 1940 et dans les années 1950, en ont eu à peu près le même nombre (autour de 2,10) mais à des âges plus jeunes.

L'âge des mères à la naissance de leur enfant, 30,7 ans en moyenne en 2017, dépasse le record observé il y a 100 ans, mais on est alors en pleine Première Guerre mondiale; l'âge des mères a augmenté de façon exceptionnelle à cette époque jusqu’à dépasser 30 ans entre 1916 et 1919 (figure 3). $\mathrm{Au}$ cours de la période récente, l'âge à la maternité n'a cessé de progresser depuis 1977, année où les femmes ont accouché en moyenne à l'âge de 26,5 ans. Elles sont mères de leur premier enfant à 24 ans en moyenne cette année-là, elles le sont près de cinq ans plus tard aujourd'hui [5].

\section{Le report des maternités, jusqu'à quels âges?}

Le mouvement de report des maternités, général dans le monde développé, est associé à l'allongement de la durée des études, à la progression de l'emploi féminin et au souhait croissant des femmes et des hommes de n'avoir des enfants qu'une fois installés dans la vie, avec des diplômes, un emploi stable, un logement et une vie de couple. La diffusion de la contraception moderne et la légalisation de l'interruption volontaire de grossesse ont contribué à ce report en réduisant la fréquence des grossesses et naissances non désirées, notamment aux âges jeunes.

Le report des maternités va-t-il se poursuivre ? L'âge moyen à la maternité pourrait continuer à augmenter en France dans les prochaines années, mais de combien? Il n'est pas exclu qu'il atteigne 32 ans, comme c'est déjà presque le cas en Espagne (31,9 ans en 2015). Mais il est peu probable qu'il augmente jusqu'à 35 ou 40 ans. La raison en est d'abord biologique. À trop attendre pour devenir mères, les femmes risquent de ne plus pouvoir enfanter quand elles le décident. Le risque moyen de ne pas avoir d'enfant croît vite avec l'âge : $4 \%$ à 20 ans, $14 \%$ à 35 ans, $35 \%$ à 40 ans et près de $80 \%$ à 45 ans [6].

L'aide médicale à la procréation, qui s'est beaucoup développée récemment, laisse espérer une solution aux couples qui ont des difficultés de conception. Mais trop peu savent que la médecine reste souvent impuissante après 40 ans. Les naissances de mères de 40 ans ou plus ne représentent qu'une fraction minime des naissances ( $4 \%$ en France en 2016). Même en hausse, elles ne devraient pas représenter une part importante des naissances, sauf à imaginer de repousser l'âge de la ménopause pour toutes les femmes, une perspective qui relève pour l'instant de la démographie-fiction.

\section{Près de $60 \%$ des enfants naissent de parents non mariés}

Parmi les 745000 enfants nés en France métropolitaine en 2016, 435000 (soit 58,5 \%) avaient des parents non mariés lorsqu'ils sont nés. Cinquante ans plus tôt, en 1966,

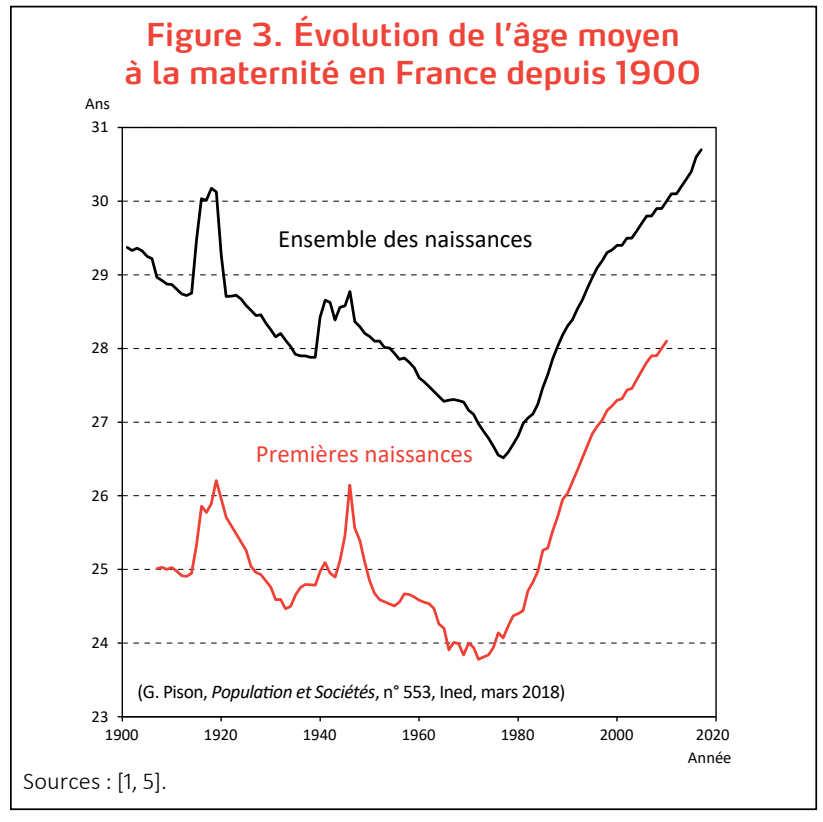

Figure 4. Évolution de la proportion de naissances hors mariage et de la proportion d'enfants non reconnus dans l'année par le père

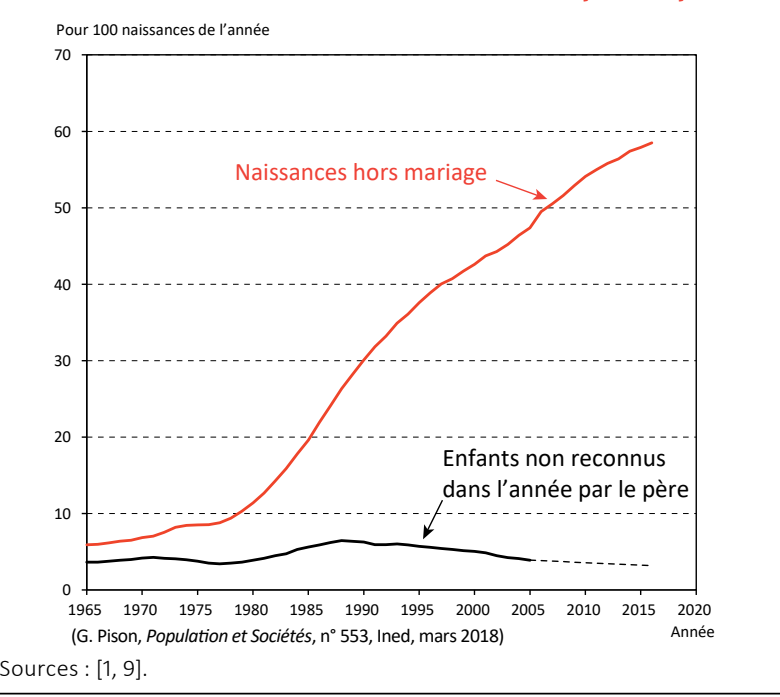

ils n'étaient que $6 \%$ dans ce cas (figure 4). Ces naissances, alors rares et mal perçues, étaient classées officiellement comme " naturelles » ou " illégitimes ». Mais la norme sociale a changé et elles sont aujourd'hui plus nombreuses que les naissances de parents mariés.

Depuis juillet 2006, la loi française ne différencie plus les droits des enfants nés de parents mariés de ceux nés de parents non mariés, et elle ne distingue plus le type de naissance selon l'état matrimonial des parents lors de l'enregistrement. Les naissances hors mariage surviennent désormais le plus souvent au sein de couples stables. Leur filiation n'est pas établie automatiquement dès la déclaration de leur naissance, comme pour les enfants nés de couples mariés ; elle nécessite dans leur cas une reconnaissance paternelle. Au début des années soixante-dix, seul un enfant né hors mariage sur cinq était reconnu par le père dès sa naissance, et en 1980 un sur deux [7, 8]. En 2005, ils sont cinq sur six à l'être. Avec la banalisation des naissances de couples non mariés, la reconnaissance paternelle 


\section{Bilan démographique 2017 : la natalité continue de baisser}

$\mathrm{Au} 1^{\mathrm{er}}$ janvier 2018, la population de la France métropolitaine est estimée à 65,0 millions d'habitants auxquels s'ajoutent 2,2 millions d'habitants des départements d'outre-mer, soit un total de 67,2 millions [1]. En métropole, la population a augmenté d'environ 217000 habitants en $2017(+0,4 \%)$ (tableau). Le solde naturel, excédent des naissances sur les décès, continue de diminuer ; il a baissé de près de moitié en sept ans, passant de 262000 (en 2010) à 138000 (en 2017). Cette baisse vient pour $60 \%$ d'une diminution des naissances et pour $40 \%$ d'une hausse des décès.

Le nombre des naissances diminue (781 000 en 2014 en France métropolitaine, 760000 en 2015, 745000 en 2016 et 728000 en 2017), sous l'effet de la diminution du nombre de femmes en âge d'avoir des enfants et de la baisse de l'indicateur conjoncturel de fécondité : 1,97 enfant par femme en 2014, 1,92 en 2015, 1,89 en 2016 et 1,85 en 2017. La diminution de cet indicateur est due à celle des taux de fécondité avant 37 ans, tandis que ceux au-delà de 37 ans augmentent légèrement. La tendance observée depuis quarante ans - avoir ses enfants de plus en plus tard-se poursuit : les femmes qui ont accouché en 2017 avaient en moyenne 30,7 ans (voir article).

L'examen de la fécondité par génération montre que les femmes nées en 1967, qui ont atteint 50 ans en 2017 et ont donc maintenant achevé leur vie féconde, ont eu en moyenne 2,02 enfants. Les femmes nées en

* L'âge moyen à la naissance du premier enfant se situe autour de 28,7 ans en 2017.

est devenue la règle et s'effectue rapidement. Au total, moins de $4 \%$ de l'ensemble des enfants ne sont pas reconnus dans l'année de leur naissance. Cette proportion est aujourd'hui plus faible qu'il y a 50 ans (figure 4) [9]. La distinction qui existait auparavant entre enfants légitimes et enfants naturels s'est déplacée : elle passe aujourd'hui entre enfants non reconnus par leur père et enfants bénéficiant de la double filiation, que leurs parents soient mariés ou non au moment de la naissance [7].

\section{Le succès du pacs : presque aussi fréquent que le mariage aujourd'hui}

Depuis l'instauration du pacte civil de solidarité (pacs) en 1999, le nombre de nouveaux pacs conclus augmente d'année en année (figure 5). Il a presque décuplé entre 2001 et 2016, passant d'environ 20000 à 192 000. L'année 2016 ayant vu la célébration de 233000 mariages, on se rapproche d'un pacs pour un mariage. Le pacs a été instauré en partie pour permettre aux couples de même sexe de faire enregistrer leur union et de bénéficier des mêmes droits que les couples de sexe différent non mariés. On pensait qu'il allait surtout être utilisé par les premiers. La surprise a été que les seconds s'en soient emparés massivement : la forte hausse du nombre de
1977, qui ont eu 40 ans en 2017, en ont déjà 1,99, soit légèrement plus que leurs aînées de 10 ans au même âge $(1,97)$, si bien que le total atteindra sans doute au moins 2,0 enfants à 50 ans pour elles aussi.

\section{L'espérance de vie augmente-t-elle moins vite?}

Le nombre de décès observés en 2017, 590 000, est supérieur de 1,5\% aux 581000 de 2016, sachant que la population a crû de $0,4 \%$ et la proportion de personnes âgées a augmenté. Le calcul de l’espérance de vie permet d'éliminer dans les fluctuations de la mortalité ce qui est dû aux variations de la taille de la population et de sa répartition par âge, pour ne faire apparaître que ce qui tient à l'évolution des risques de décès. L'espérance de vie à la naissance atteint 79,5 ans pour les hommes et 85,4 ans pour les femmes en 2017 , contre 79,3 ans et 85,3 ans en 2016, soit un gain de 0,2 an pour les hommes et 0,1 an pour les femmes. Ce gain est modeste, comme ceux des années précédentes : l'espérance de vie était de 78,0 ans pour les hommes et 84,7 ans pour les femmes en 2010 , et n'a donc gagné depuis que 1,5 an pour les hommes et 0,7 an pour les femmes, soit en moyenne annuelle 0,2 an et moins de 0,1 an. C'est moins que la tendance des cinquante dernières années : trois mois par an (0,25 an) (figure 1 ; article). Les progrès de l'espérance de vie seraient-ils en train de s'essouffler ? Il est probablement encore trop tôt pour conclure dans ce sens ou annoncer qu'elle a atteint ses limites.

pacs vient principalement de l'essor des pacs entre personnes de sexe différent. Leur nombre est passé de 15000 à 184000 , soit une multiplication par douze en quinze ans. Les pacs entre personnes de même sexe n'ont fait que doubler dans l'intervalle, passant de près de 3500 à un peu plus de 7000 par an. Ils représentent aujourd'hui $4 \%$ de l'ensemble des pacs.

L'essor du pacs, alors que le nombre de mariages a diminué dans le même temps (passant de 296000 à 233000 entre 2001 et 2016, soit une baisse de 63 000), amène à se demander si le pacs ne serait pas en train de remplacer progressivement le mariage?

Les deux types d'union se sont beaucoup rapprochés ces dernières années : le régime fiscal est devenu similaire en 2005, et le nouveau couple "pacsé » bénéficie depuis d'une imposition commune sur les revenus dès la première année du pacte, comme le nouveau couple marié, alors qu'il devait auparavant attendre trois années de vie commune. Les nouveaux pacsés ont eu la possibilité, comme les nouveaux mariés, de faire trois déclarations de revenus dans l'année de leur union, deux séparées jusqu’à la date de l'union et une commune pour le restant de l'année. Cette disposition fiscale, avantageuse notamment pour les couples avec deux revenus, a été supprimée à partir de 2011 


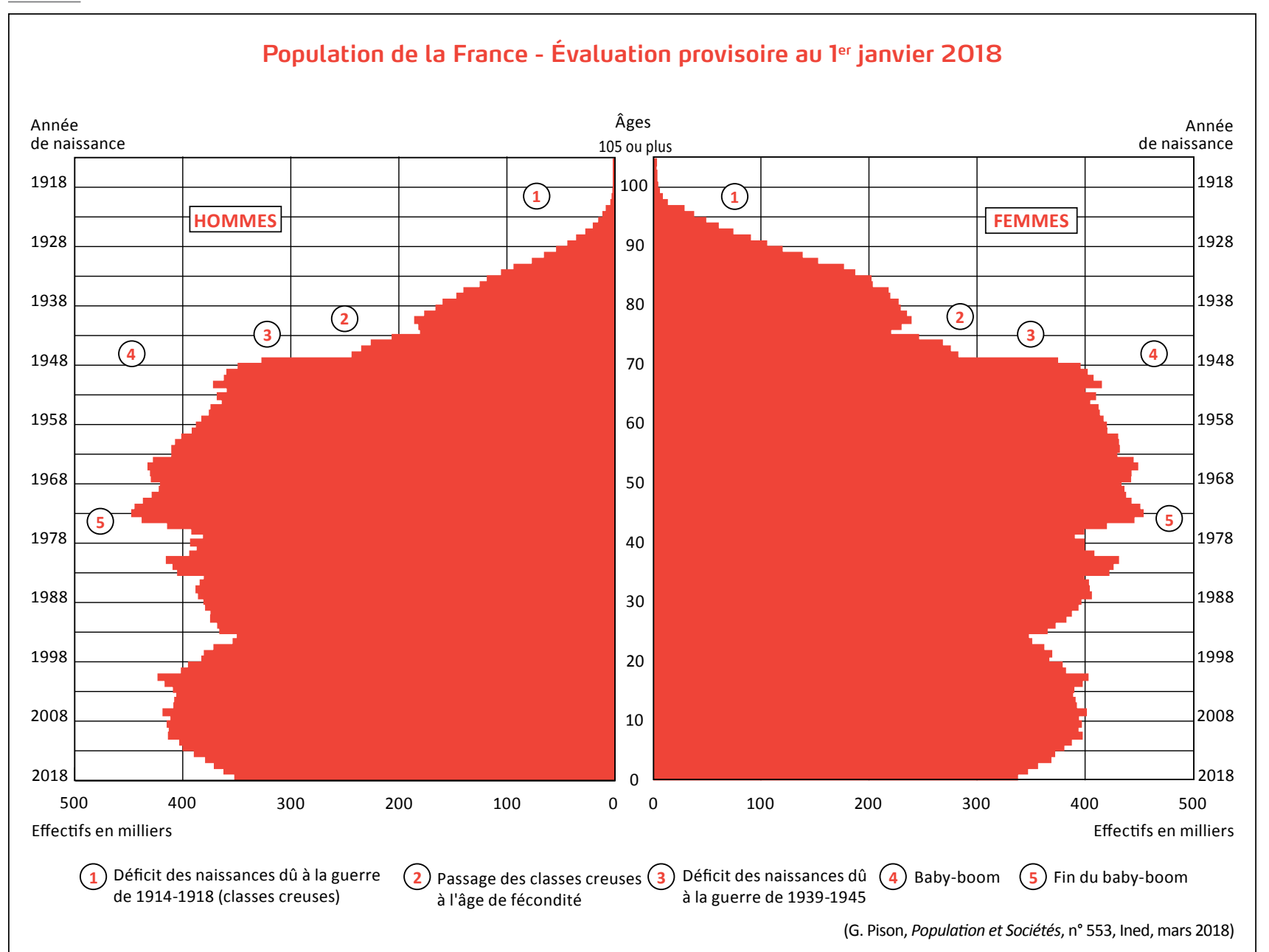

(G. Pison, Population et Sociétés, $n^{\circ} 553$, Ined, mars 2018)

\begin{tabular}{|c|c|c|c|c|c|c|c|c|c|c|c|c|c|c|c|}
\hline \multicolumn{16}{|c|}{ Tableau - Indicateurs démographiques 1950 à 2017, France métropolitaine } \\
\hline & 1950 & 1960 & 1970 & 1980 & 1990 & 2000 & 2009 & 2010 & 2011 & 2012 & 2013 & 2014 & 2015(p) & $2016(p)$ & 2017(p) \\
\hline Naissances (m) & 858 & 816 & 848 & 800 & 762 & 775 & 793 & 802 & 793 & 790 & 782 & 781 & 760 & 745 & 728 \\
\hline Décès (m) & 530 & 517 & 540 & 547 & 526 & 531 & 538 & 540 & 535 & 559 & 558 & 547 & 582 & 581 & 590 \\
\hline Excédent naturel $(\mathrm{m})$ & 328 & 299 & 308 & 253 & 236 & 244 & 255 & 262 & 258 & 231 & 223 & 234 & 179 & 164 & 138 \\
\hline Solde migratoire $(\mathrm{m})$ & 35 & 140 & 180 & 44 & 80 & 70 & 44 & 43 & 47 & 91 & 107 & 39 & 79 & 79 & 79 \\
\hline Variation totale $(\mathrm{m})$ & 363 & 439 & 488 & 297 & 316 & 314 & 299 & 305 & 305 & 322 & 331 & 273 & 257 & 243 & 217 \\
\hline Ajustement ${ }^{(1)}(\mathrm{m})$ & - & - & - & - & - & 94 & - & - & - & - & - & - & - & - & - \\
\hline Taux de natalité ( $\mathrm{t}$ ) & 20,5 & 17,9 & 16,7 & 14,9 & 13,4 & 13,1 & 12,7 & 12,7 & 12,5 & 12,4 & 12,2 & 12,2 & 11,8 & 11,5 & 11,2 \\
\hline Taux de mortalité (t) & 12,7 & 11,3 & 10,6 & 10,2 & 9,3 & 9,0 & 8,6 & 8,6 & 8,5 & 8,8 & 8,7 & 8,5 & 9,0 & 9,0 & 9,1 \\
\hline Taux de mort. infantile $(r)$ & 51,9 & 27,4 & 18,2 & 10,0 & 7,3 & 4,4 & 3,7 & 3,5 & 3,3 & 3,3 & 3,5 & 3,3 & 3,5 & 3,5 & 3,6 \\
\hline Indice de fécondité (e) & 2,93 & 2,73 & 2,47 & 1,94 & 1,78 & 1,87 & 1,99 & 2,02 & 2,00 & 1,99 & 1,97 & 1,97 & 1,92 & 1,89 & 1,85 \\
\hline Espérance de vie: & & & & & & & & & & & & & & & \\
\hline hommes (a) & 63,4 & 67,0 & 68,4 & 70,2 & 72,7 & 75,3 & 77,8 & 78,0 & 78,4 & 78,5 & 78,8 & 79,3 & 79,0 & 79,3 & 79,5 \\
\hline femmes (a) & 69,2 & 73,6 & 75,9 & 78,4 & 80,9 & 82,8 & 84,5 & 84,7 & 85,0 & 84,8 & 85,0 & 85,4 & 85,1 & 85,3 & 85,4 \\
\hline Mariages ${ }^{(2)}(\mathrm{m})$ & 331 & 320 & 394 & 334 & 287 & 298 & 245 & 245 & 231 & 240 & 233 & 235 & 230 & 227 & 223 \\
\hline Taux de nuptialité (t) & 7,9 & 7,0 & 7,8 & 6,2 & 5,1 & 5,0 & 3,9 & 3,9 & 3,7 & 3,8 & 3,6 & 3,7 & 3,6 & 3,5 & 3,4 \\
\hline Population ${ }^{(3)}(\mathrm{m})$ & 42010 & 45904 & 51016 & 54029 & 56893 & 59267 & 62765 & 63070 & 63376 & 63698 & 64028 & 64301 & 64558 & 64801 & 65018 \\
\hline Moins de 20 ans ${ }^{(2)}(\mathrm{m})$ & 12556 & 14665 & 16748 & 16419 & 15632 & 15068 & 15407 & 15440 & 15458 & 15513 & 15589 & 15652 & 15683 & 15690 & 15688 \\
\hline 65 ans ou plus ${ }^{(2)}(\mathrm{m})$ & 4727 & 5288 & 6174 & 7541 & 8036 & 9561 & 10540 & 10667 & 10973 & 11302 & 11649 & 11989 & 12309 & 12593 & 12879 \\
\hline Moins de 20 ans ${ }^{(2)} \%$ & 29,9 & 31,9 & 32,8 & 30,4 & 27,5 & 25,4 & 24,5 & 24,5 & 24,4 & 24,4 & 24,3 & 24,3 & 24,3 & 24,2 & 24,1 \\
\hline 65 ans ou plus ${ }^{(2)} \%$ & 11,3 & 11,5 & 12,1 & 14,0 & 14,1 & 16,1 & 16,8 & 16,9 & 17,3 & 17,7 & 18,2 & 18,6 & 19,1 & 19,4 & 19,8 \\
\hline \multicolumn{16}{|c|}{$\begin{array}{l}\text { (a) années - (e) nombre d'enfants par femme - (m) milliers - (p) provisoire - (r) pour } 1000 \text { naissances vivantes - (t) pour } 1000 \text { habitants. } \\
\text { (1) les estimations de population pour l'année } 2000 \text { tiennent compte d'un ajustement destiné à rétablir la cohérence comptable entre les recensements de } 1999 \\
\text { et } 2006 \text { (voir Sylvain Papon et Catherine Beaumel, } 2018 \text { [1]). } \\
\text { (2) y compris mariages de personnes de même sexe à partir de } 2013 . \\
\text { (3) en fin d'année. } \\
\text { Source : Insee, Division des enquêtes et études démographiques (http://www.insee.fr). }\end{array}$} \\
\hline
\end{tabular}


Figure 5. Évolution du nombre annuel de nouvelles unions (mariages et pacs) depuis 1965 Effectif (en milliers)

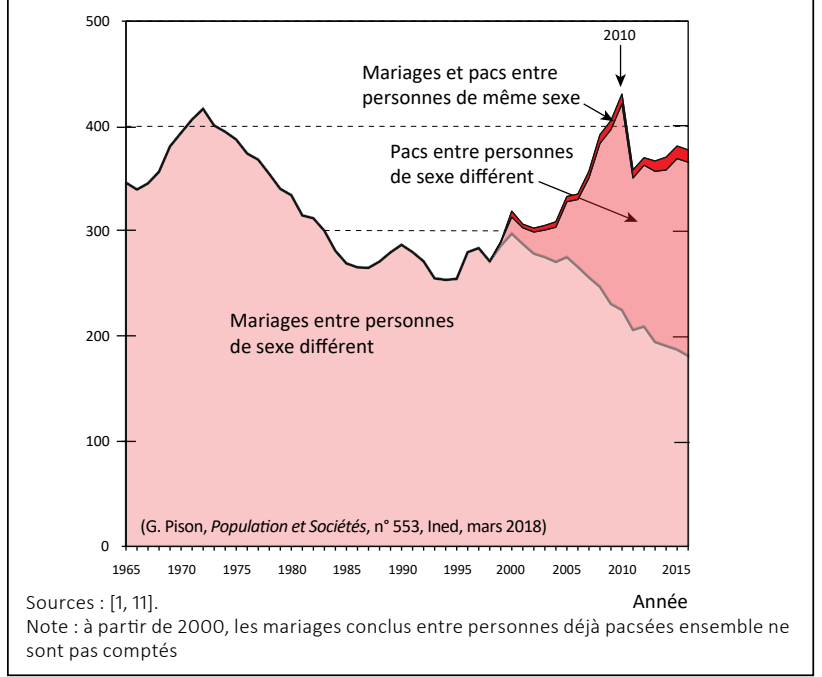

à la fois pour le pacs et le mariage. Il en a résulté un pic de pacs la dernière année du dispositif, en 2010 (figure 5). Rien de pareil pour les mariages, révélant que si une partie des pacs sont conclus pour de simples raisons juridiques ou fiscales, ce n'est pas le cas pour la plupart des mariages.

L'âge moyen des nouveaux pacsés hétérosexuels, 30,4 ans pour les femmes en 2005 et 32,4 ans pour les hommes, est très proche de celui des personnes se mariant pour la première fois : 29,7 ans pour les femmes et 31,9 ans pour les hommes. La fréquence des ruptures est également voisine : près d'un pacs hétérosexuel sur cinq est rompu au bout de six ans $(18,9 \%)$, contre près d'un mariage sur $\operatorname{sept}(13,5 \%)[10]$.

L'ouverture du mariage aux personnes de même sexe en 2013 n'a pas modifié les tendances. Il y a eu autant de mariages que de pacs conclus entre personnes de même sexe en 2016 (respectivement 7113 et 7 112), sachant qu'une partie des mariages (36 \% en 2016) unissait des personnes déjà pacsées auparavant.

On peut suivre l'évolution des nouvelles unions enregistrées, quelle qu'en soit la forme (pacs ou mariage) en cumulant les nombres annuels de pacs et de mariages ${ }^{1}$ (figure 5). Le nombre total de nouvelles unions croît régulièrement depuis le milieu des années 1990, puisque la diminution des mariages depuis 2000 est plus que compensée par l'augmentation des pacs.

Les dernières décennies ont aussi vu les divorces devenir plus fréquents, suivis pour partie de remises en couple, conduisant à la montée des familles monoparentales et

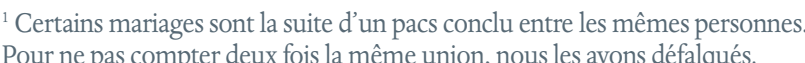

recomposées. Avecl'allongement de la vie, l'union de toute une existence fait place à plusieurs couples successifs pour une part croissante des individus.

Il y a cinquante ans, notamment en 1968, l'idée que le couple et la famille avaient fait leur temps était répandue. Force est de constater que non seulement ils n'ont pas disparu, mais que la proportion d'individus vivant en couple est restée à peu près la même, ainsi que celles des personnes enregistrant leur union. Elle a même augmenté ces vingt dernières années grâce à l'offre de nouvelles formes d'unions.

\section{RÉFÉRENCES}

[1] Sylvain Papon et Catherine Beaumel- « Bilan démographique 2017 : plus

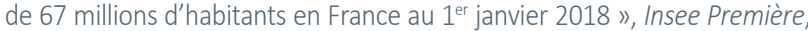
$n^{\circ} 1683,2018$.

[2] Gilles Pison- « France 2004 : I'espérance de vie franchit le seuil de 80 ans ». Population et Sociétés, $n^{\circ}$ 410, 2005, 4 p.

[3] France Meslé et Jacques Vallin - «La transition sanitaire : tendances et perspectives », in Graziella Caselli, Jaques Vallin et Guillaume Wunsch (dir.), Démographie : analyse et synthèse, t. 3, Ined, 2002, p. 439-461.

[4] Jacques Vallin et France Meslé- Tables de mortalité françaises pour les $X I X^{e}$ et $X X^{e}$ siècles et projections pour le XXe siècle, Données et statistiques, $n^{\circ}$ 4-2001, Ined, 2001, $101 \mathrm{p}$.

[5] Didier Breton, Magali Barbieri, Hippolyte d'Albis, Magali Mazuy " L'évolution démographique récente de la France : de forts contrastes départementaux », Population, 72(4), 2017, p. 583-652.

[6] Henri Leridon- «A new estimate of permanent sterility by age: Sterility defined as the inability to conceive », Population Studies, 62(1), 2008, p. 15-24. [7] Catherine Beaumel, Roselyne Kerjosse et Laurent Toulemon - « Des mariages, des couples et des enfants ॥, Insee Première, 1999, n 624.

[8] Francisco Muñoz-Pérez et France Prioux - " Naître hors mariage », Population et Sociétés, $\mathrm{n}^{\circ}$ 342, 1999.

[9] Laurent Toulemon - " Les pères dans les statistiques », Informations sociales, $n^{\circ} 176,2013$, p. 8-13

[10] Valérie Carrasco - « Le pacte civil de solidarité : une forme d'union qui se banalise », Infostat-Justice, $n^{\circ}$ 97, 2007.

[11] Ministère de la Justice - « Statistiques des pactes civils de solidarité », http://www.justice.gouv.fr/statistiques-10054/donnees-statistiques-10302/ pactes-civils-de-solidarite-pacs-25129.html

\section{Résumé}

L'espérance de vie a augmenté de 11 ans en France entre 1967 et 2017, passant de 71,5 ans à 82,5 ans sexes confondus. Une telle progression dépasse toutes les prévisions, et l'espérance de vie n'a toujours pas atteint de plafond comme on le prévoyait il y a cinquante ans. Trois autres surprises démographiques ont marqué le demisiècle passé : le report des naissances, avec un recul de l'âge moyen à la maternité de plus de 4 ans depuis 1977, l'augmentation des naissances hors mariage, qui sont désormais majoritaires, et l'invention et le succès du pacs, les nouveaux pacs conclus étant presque aussi fréquents que les mariages aujourd'hui.

\section{Mots-clés}

Population, France, espérance de vie, âge à la maternité, naissances hors mariage, pacs, 1968-2018
Ined : 133, boulevard Davout, 75980 Paris, Cedex 20 Directrice de la publication : Magda Tomasini Rédacteur en chef : Gilles Pison Éditrice : Marie-Paule Reydet Graphiste : Isabelle Milan

Impression : Mérico Delta Print, Bozouls, France D. L. 1er trim. $2018 \cdot$ ISSN 01847783 D. L. ler trim. $2018 \cdot$ ISSN 01847783

INSTITUT NATIONAL Numéro 553 - mars 2018 - Population \& Sociétés. D'ÉT U D E S bulletin mensuel d'information de l'Institut national DÉMOGRA
PHIQUES d'études démographiques
Retrouvez Population et Sociétés

dès sa parution sur le site internet de l'Ined et abonnez-vous :

www.ined.fr/fr/publications/populationet-societes

Contact : edition@ined.fr

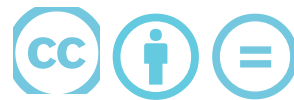

Cet article peut être reproduit sur papier ou en ligne gratuitement en utilisant notre licence Creative Commons 